\title{
The Intradermal Leprolin Test for the Detection of Early and Latent Leprosy.
}

\section{LEONARD ROGERS.}

$\mathbf{N}$

OW that the solution of the leprosy problem depends mainly on the early diagnosis of infections, especially among the households and other contacts of infective cases, the intradermal leprolin test of $\mathrm{P}$. Bargehr, which has been confirmed by C. D. de Langen and W. de Vogel, deserves to be more widely known and utilised. Leprolin was made by Bargehr by mincing lepra nodules containing large numbers of the bacilli in a little water, heating on a water-bath at $100^{\circ} \mathrm{C}$. for twenty minutes, and adding $\frac{1}{2}$ per cent. carbolic acid, but de Langen prefers to keep lepromatous emulsions in dry powdered form in sealed ampoules after sterilising at $120^{\circ} \mathrm{C}$.

The test is carried out by scarifying two small areas on the upper arm and lightly rubbing a little of the leprolin into one of them for five minutes and allowing it to dry for one hour ; the other area is kept as a control. A positive reaction consists in the appearance of a slightly raised erythematous patch ; this appears in one to seven days, and may last up to five or six weeks. It is not every leprous nodule which yields a suitable preparation of antigen, and de Vogel states that it is not easy to obtain a suitable one, so it is necessary to obtain a reliable preparation from a well-equipped laboratory, such as that of E. Muir, in the Calcutta School of Tropical Medicine.

The principle on which the reaction is based is that patients with active leprosy infections, whose tissues are accustomed to the influence of the toxins of the lepra bacilli, give negative results on the application of leprolin to the scarified skin; but those who have never been in contact with the disease, as well as old "burnt out" nerve cases of leprosy with no active symptoms for some years, give positive reactions. Some of those who have been in contact with lepers for long are also found to give negative reactions. This indicates that they have become infected at some time or other, and they may either be in the incubation stage of the disease, or they may have developed immunity through a mild infection without showing active symptoms, as with tuberculosis. Very early cases of the disease give negative reactions ; these may be of diagnostic value. For example, Bargehr reports on 168 tests, in 82 of which lepra bacilli 
were found in the tissues of the patients and all were negative, while forty-five out of eighty patients in whom no lepra bacilli were found also gave negative reactions, and most of these showed clear symptoms of leprosy. The thirty-five positive reactions included persons with no signs of leprosy, and also some with healed mutilated fingers and toes of nerve cases of leprosy, who were in good general health and had shown no change for several years. De Vogel records tests in nine family groups of whom only the healthy reacted, with the exception of two who will be watched for the appearance of early symptoms. Further, some very early cases gave negative reactions of diagnostic value. Professor Lyle Cummins has recently tried subcutaneous injections of leprolin supplied by E. Muir in a number of lepers and others in South Africa, but the results were irregular, so the intradermal method appears to be the better one.

\section{Application of the Test in the Early Diagnosis of Leprosy Contacts.}

I have repeatedly pointed out that the rapid reduction of leprosy depends on the frequent examination of the household and other close contacts of all known lepers, because about 90 per cent. of new cases are likely to be found among them, and the great majority of these could be cleared up before they had reached an infective stage, if detected by such examinations of contacts and treated in the earliest stages of the disease. This theory has now been demonstrated to be true by the work of Dr. George Bray under very favourable conditions in the small island of Nauru, in Oceana, for by means of a monthly examination of all the small population for the first signs of leprosy, the segregation of the infective cases on one side of the island, and the regular treatment of the bacteriologically negative ones as outpatients without isolation, a very heavy incidence of the disease has been reduced by about one-third in three years. Moreover, no early treated case has gone on to the advanced highly infective nodular stage. Under such carefully controlled conditions the intradermal leprosy test promises to be of great value if used in the following manner, and it should also furnish important scientific data.

All close contacts, who have lived in the same house with a leper, should be tested, and all who give negative reactions should be looked on as having been infected at some time or other. They may either be in the incubation period, or possibly they may have passed through a mild unrecognised 
infection and developed immunity, but further experience is required on these points. I therefore suggest that one-half of such negatively reacting cases should be treated for leprosy by injections for six months or so, with a view to clearing them of infection while still in the incubation stage, and so preventing the appearance of definite symptoms. The remaining half should be kept as controls, and watched very carefully for the first signs of the disease before being submitted to treatment. The negatively reacting cases should be retested after six months or more treatment, as the appearance of a positive reaction might indicate that they had been cured of a latent infection. If experience of the test should show that the control negatively reacting cases develop signs of the disease in definitely larger proportions than those who are treated in the incubation stage, the value of the test will be so evident that all such incubation period cases should be treated without delay. In that event, a further aid of great practical importance in the campaign against leprosy will be established. Bargehr also suggests five to seven intradermal leprolin scarifications may possibly produce immunity against leprosy, but this remains to be proved. 